\title{
Solving of Linear Volterra-Fredholm Integral Equations via Modification of Block Pulse Functions
}

\author{
AyYubi Ahmad \\ Department of Mathematics, Faculty of Applied Science and Technology, Ahmad \\ Dahlan University, \\ Jl. Ringroad Selatan, Kragilan, Tamanan, Banguntapan, Bantul, Yogyakarta 55191, \\ Indonesia, Email: ayyubiahmad1@gmail.com
}

\begin{abstract}
A computational method based on modification of block pulse functions is proposed for solving numerically the linear Volterra-Fredholm integral equations. We obtain integration operational matrix of modification of block pulse functions on interval $[0, T)$. A modification of block pulse functions and their integration operational matrix can be reduced to a linear upper triangular system. Then, the problem under study is transformed to a system of linear algebraic equations which can be used to obtain an approximate solution of linear Volterra-Fredholm integral equations. Furthermore, the rate of convergence is $\mathcal{O}(h)$ and error analysis of the proposed method are investigated. The results show that the approximate solutions have a good of efficiency and accuracy.

Keywords: Integration Operational Matrix, Linear Volterra-Fredholm Integral Equations, $\varepsilon$ MBPFs.
\end{abstract}

\section{Introduction}

An integral equation is defined as an equation in which the unknown function $X(t)$ to be determined appear under one or more integral signs. The subject of integral equations is one of the most useful mathematical tools in pure and applied mathematics. It arise naturally in physics, chemistry, biology, and engineering applications modelled by initial value problems for a finite interval $[a, b]$. It also arise as representation formulas for the solutions of differential equations. Indeed, a differential equation can be replaced by an integral equation that incorporates its boundary conditions [4]. It has enormous applications in many physical problems. Many initial and boundary value problems associated with ordinary differential equation (ODE) and partial differential equation (PDE) can be transformed into problems of solving some approximate integral equations [8].

The Volterra-Fredholm integral equation, which is a combination of disjoint Volterra and Fredholm integrals, appears in one integral equation. The Volterra-Fredholm integral equations arise from parabolic boundary value problems, mathematical modelling of the spatiotemporal development of an epidemic, various physical, biological, and chemical applications $[10,11]$. There are several techniques for approximating the solution such as moving least square method and Chebyshev polynomials [1], collocation and Galerkin methods [2], parameterized pseudospectral integration matrices [9], triangular functions [5], Taylor polynomial [12],

2000 Mathematics Subject Classification: 45D05

Submitted: 2021-02-12, Revisions Required: 2021-04-13, Accept Submission: 2021-05-29 
and Legendre collocation method [7]. In this paper, we apply a numerical method based on the modification of block pulse functions and integration operational matrix to consider the following linear Volterra-Fredholm integral equation:

$$
X(t)=f(t)+\int_{0}^{t} K_{1}(s, t) X(s) d s+\int_{\alpha}^{\beta} K_{2}(s, t) X(s) d s, \quad t \in[0, T),
$$

where $X(t)$ is the unknown function, $f(t)$ is analytic function, while $K_{1}(s, t)$ and $K_{2}(s, t)$ are the kernels of $L^{2}$ functions. In order to obtain an approximate solution for Eq. (1) based on modification of block pulse functions, we derive a new integration operational matrix and reduce our problem to solving a system of linear algebraic equations. Moreover, a new technique for computation of the linear terms in such equations is presented. Furthermore, convergence analysis of modification of block pulse functions is investigated. We also demonstrate the efficiency and accuracy of the proposed method.

\section{Materials and Methods}

2.1. Definition of Modified Block Pulse Functions. A set of $\varepsilon$ modified block pulse functions $(\varepsilon \mathrm{MBPFs}) \psi_{i}(t), i=0,1, m$ on the interval $[0, T)$ are defined as

$$
\begin{gathered}
\psi_{0}(t)= \begin{cases}1, & t \in[0, h-\varepsilon)=I_{0}, \\
0, & \text { otherwise, }\end{cases} \\
\psi_{i}(t)= \begin{cases}1, & t \in[i h-\varepsilon,(i+1) h-\varepsilon)=I_{i}, \\
0, & \text { otherwise, }\end{cases}
\end{gathered}
$$

for $i=1,2, \ldots, m-1$ and

$$
\psi_{m}(t)=\left\{\begin{array}{lc}
1, & t \in[T-\varepsilon, T)=I_{m} \\
0, & \text { otherwise }
\end{array}\right.
$$

with a positive integer value for $m$ and $h=\frac{T}{m}$.

2.2. Properties of MBPFs. The important properties of $\varepsilon$ MBPFs are as follows

- Disjointness:

$$
\psi_{i}(t) \psi_{j}(t)=\left\{\begin{array}{cc}
\psi_{i}(t), & i=j \\
0, & i \neq j
\end{array}\right.
$$

where $i, j=0, \ldots, m$.

- Orthogonality:

$$
\int_{0}^{T} \psi_{i}(t) \psi_{j}(t) d t=h \delta_{i j}
$$

where $i, j=1, \ldots, m-1$ and $\delta_{i j}$ is Kronecker delta.

- Completeness:

$$
\int_{0}^{T} f^{2}(t) d t=\sum_{i=0}^{\infty} f_{i}^{2}\left\|\psi_{i}(t)\right\|^{2}
$$

where

$$
f_{i}=\frac{1}{\Delta\left(l_{i}\right)} \int_{0}^{T} f(t) \psi_{i}(t) d t
$$

and $\Delta\left(l_{i}\right)$ is length of interval $I_{i}$ 
2.3. Function Approximation. Rewriting Eq. (2) in the vector form we have

$$
f(t) \simeq \sum_{i=0}^{m} f_{i} \psi_{i}(t)=F^{T} \Psi(t)=\Psi^{T}(t) F,
$$

in which

$$
F=\left(\begin{array}{llll}
f_{0} & f_{1} & \ldots & f_{m}
\end{array}\right)^{T}
$$

and

$$
\Psi(t)=\left(\begin{array}{llll}
\psi_{0}(t) & \psi_{1}(t) & \ldots & \psi_{m}(t)
\end{array}\right)^{T}
$$

Moreover, any two dimensional function $k(s, t) \in L^{2}\left(\left[0, T_{1}\right) \times\left[0, T_{2}\right)\right)$ can be expanded with respect to $\varepsilon \mathrm{MBPFs}$ such as

$$
k(s, t) \simeq \Psi^{T}(s) K \Psi(t)=\Psi^{T}(t) K^{T} \Psi(s),
$$

where $\Psi(s)$ and $\Psi(t)$ are $m_{1}$ and $m_{2}$ dimensional $\varepsilon$ MBPFs vectors respectively, and $K=$ $\left(k_{i j}\right), i=0,1, \ldots, m_{1}, j=0,1, \ldots, m_{2}$ is the $m_{1} \times m_{2} \varepsilon$ modified block pulse coefficient matrix with

$$
k_{i j}=\frac{1}{\Delta\left(I_{i}\right) \Delta\left(I_{j}\right)} \int_{0}^{T_{1}} \int_{0}^{T_{2}} k(s, t) \Psi_{i}(s) \Psi_{j}(t) d t d s .
$$

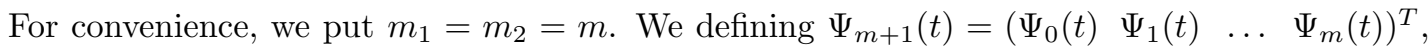
we have

$$
\Psi_{m+1}(t) \Psi_{m+1}^{T}(t)=\left(\begin{array}{cccc}
\psi_{0}(t) & 0 & \ldots & 0 \\
0 & \psi_{1}(t) & \cdots & 0 \\
\vdots & \vdots & \ddots & \vdots \\
0 & 0 & \ldots & \psi_{m}(t)
\end{array}\right)_{(m+1) \times(m+1)}
$$

Furthermore

$$
\Psi_{m+1}^{T}(t) \Psi_{m+1}(t)=1
$$

and

$$
\Psi_{m+1}(t) \Psi_{m+1}^{T}(t) F=D_{F} \Psi_{m+1}(t),
$$

where $D_{F}$ usually denotes a diagonal matrix whose diagonal entries are related to a constant vector $F=\left(\begin{array}{llll}f_{0} & f_{1} & \ldots & f_{m}\end{array}\right)^{T}$.

2.4. Intergration Operational Matrix. Similar to block pulse functions,

$$
\int_{0}^{t} \Psi_{m+1}(s) d s \simeq Q \Psi_{m+1}(t)
$$

where the integration operational matrix $Q$ of $\varepsilon$ MBPFs is given by

$$
Q=\left(\begin{array}{cccc}
\frac{h-\varepsilon}{2} & h-\varepsilon & \ldots & h-\varepsilon \\
0 & \frac{h}{2} & \ldots & h \\
\vdots & \vdots & \ddots & \vdots \\
0 & 0 & \ldots & \frac{\varepsilon}{2}
\end{array}\right)_{(m+1) \times(m+1)}
$$

So, the integral of every function $f(t)$ can be approximated as follows

$$
\int_{0}^{t} f(s) d s \simeq \int_{0}^{t} F^{T} \Psi_{m+1}(s) d s \simeq F^{T} Q \Psi_{m+1}(t) .
$$




\section{Result And Discussion}

3.1. Solving Volterra-Fredholm Integral Equations by Modification of Block Pulse Functions. We consider following linear Volterra-Fredholm integral equation

$$
X(t)=f(t)+\int_{\alpha}^{\beta} K_{1}(s, t) X(s) d s+\int_{0}^{t} K_{2}(s, t) X(s) d s, \quad t \in[0, T) .
$$

We approximate functions $X(t), f(t), k_{1}(s, t)$, and $k_{2}(s, t)$ by $\varepsilon$ MBPFs as follows

$$
\begin{gathered}
X(t) \simeq \Phi^{T}(t) W=W^{T} \Phi(t), \\
f(t) \simeq \Phi^{T}(t) F=F^{T} \Phi(t), \\
K_{1}(s, t) \simeq \Phi^{T}(s) K_{1} \Phi(t)=\Phi^{T}(t) K_{1}^{T} \Phi(s), \\
K_{2}(s, t) \simeq \Phi^{T}(s) K_{2} \Phi(t)=\Phi^{T}(t) K_{2}^{T} \Phi(s) .
\end{gathered}
$$

In the above approximation, $\mathrm{W}$ and $\mathrm{F}$ are modified block pulse coefficients vector, $K_{1}$ and $K_{2}$ are modified block pulse coefficients matrix.

Substituting above approximation in Eq. (3), we get

$$
W^{T} \Phi(t) \simeq F^{T} \Phi(t)+W^{T}\left(\int_{\alpha}^{\beta} \Phi(s) \Phi^{T}(s) d s\right) K_{1} \Phi(t)+W^{T}\left(\int_{0}^{t} \Phi(s) \Phi^{T}(s) d s\right) K_{2} \Phi(t) .
$$

Let $K_{j}^{i}$ be the ith row of the constant matrices $K_{j}, j=1,2,3 . R^{i}$ be the ith row of the integration operational matrix $Q, D_{K_{j}^{i}}$ be diagonal matrices with $K_{j}^{i}$ as its diagonal entries. By the relation $\int_{\alpha}^{\beta} \Phi(s) \Phi^{T}(s) d s=h I_{\left(m_{1}+1\right) \times\left(m_{2}+1\right)}$ and assuming $m_{1}=m_{2}=m$, we have

$$
\left(\int_{\alpha}^{\beta} \Phi(s) \Phi^{T}(s) d s\right) K_{1} \Phi(t)=h I K_{1} \Phi(t)=B_{1} \Phi(t),
$$

where $B_{1}=h I K_{1}=h K_{1}$.

Furthermore,

$$
\begin{aligned}
\left(\int_{0}^{t} \Phi(s) \Phi^{T}(s) d s\right) K_{2} \Phi(t)= & \left(\begin{array}{cccc}
R^{0} \Phi(t) & 0 & \ldots & 0 \\
0 & R^{1} \Phi(t) & \cdots & 0 \\
\vdots & \vdots & \ddots & \vdots \\
0 & 0 & \ldots & R^{m} \Phi(t)
\end{array}\right)\left(\begin{array}{c}
K_{2}^{0} \\
K_{2}^{1} \\
\vdots \\
K_{2}^{m}
\end{array}\right) \Phi(t) \\
& =\left(\begin{array}{c}
R^{0} \Phi(t) K_{2}^{0} \Phi(t) \\
R^{1} \Phi(t) K_{2}^{1} \Phi(t) \\
\vdots \\
R^{m} \Phi(t) K_{2}^{m} \Phi(t)
\end{array}\right) \\
& =\left(\begin{array}{c}
R^{0} \Phi(t) \Phi(t)^{T} K_{2}^{0^{T}} \\
R^{1} \Phi(t) \Phi(t)^{T} K_{2}^{1 T} \\
\vdots \\
R^{m} \Phi(t) \Phi(t)^{T} K_{2}^{m T}
\end{array}\right) \\
& =\left(\begin{array}{c}
R^{0} D_{K_{2}^{0}} \\
R^{1} D_{K_{2}^{1}}^{1} \\
\vdots \\
R^{m} D_{K_{2}^{m}}
\end{array}\right) \Phi(t)=B_{2} \Phi(t),
\end{aligned}
$$


where

$$
B_{2}\left(\begin{array}{cccc}
k_{00}\left(\frac{h-\varepsilon}{2}\right) & k_{01}(h-\varepsilon) & \ldots & k_{0 m} h-\varepsilon \\
0 & k_{11}\left(\frac{h}{2}\right) & \ldots & k_{1 m}(h) \\
\vdots & \vdots & \ddots & \vdots \\
0 & 0 & \ldots & k_{m m}\left(\frac{\varepsilon}{2}\right)
\end{array}\right)_{(m+1) \times(m+1)}
$$

with substituting (5) and (6) in (4), we get

$$
W^{T} \Phi(t) \simeq F^{T} \Phi(t)+W^{T} B_{1} \Phi(t)+W^{T} B_{2} \Phi(t) .
$$

Then,

$$
W^{T}\left(I-B_{1}-B_{2}\right) \simeq F^{T}
$$

So, by getting $N=\left(I-B_{1}-B_{2}\right)^{2}$ and replacing $\simeq$ by $=$, we have

$$
N W=F \text {. }
$$

Which is a linear system of equations with upper triangular coefficients matrix that gives the approximate modified block pulse coefficient of the unknown $X(t)$.

3.2. Error Analysis. In the following theorems, for simplicity we assume $T=1$ and $h=\frac{1}{m}$.

Theorem 3.1. If $\hat{f}_{m}(t)=\sum_{i=0}^{m} f_{i} \psi_{i}(t)$ and $f_{i}=\frac{1}{\Delta\left(I_{i}\right)} \int_{0}^{1} f(t) \psi_{i}(t) d t, i=0, \ldots, m$ then:

$i \delta=\int_{0}^{1}\left(f(t)-\sum_{i=0}^{m} f_{i} \psi_{i}(t)\right)^{2} d t$, achieves its minimum value.

ii $\left\{\hat{f}_{m}(t)\right\}$ approaches $f(t)$ pointwise.

iii $\int_{0}^{1} f^{2}(t) d t=\sum_{i=0}^{\infty} f_{i}^{2}\left\|\psi_{i}\right\|^{2}$.

Proof. Proof is like similar theorem in [3] but intervals of integration have to redefine as $I_{i}, i=0, \ldots, m$ in $(3.1)$

Theorem 3.2. Assume:

$i f(t)$ is continuous and differentiable in $[-h, 1+h]$ with bounded derivative, that is $\left|f^{\prime}(t)\right|<$ $M$.

ii $\hat{f}_{\frac{i h}{k}}(t), i=0, \ldots, k-1$, are correspondingly BPFs, $\frac{h}{k} M B P F s, \ldots, \frac{(k-1) h}{k}$ MBPFs expansions of $f(t)$ base on $m+1 \varepsilon M B P F s$ over interval $[0,1)$.

iii $\bar{f}(t)=\frac{1}{k} \sum_{i=0}^{k-1} \hat{f}_{\frac{i h}{k}}(t)$.

Then

$$
\left\|f(t)-\hat{f}_{\frac{i h}{k}}(t)\right\|=\mathcal{O}(h), \text { and }\|f(t)-\bar{f}(t)\|=\mathcal{O}\left(\frac{h}{k}\right) \text { in }[h, 1-h] .
$$

PRoOF. Trapezoidal rule for integral is

$$
\begin{gathered}
\int_{a}^{b} f(t) d t=\frac{b-a}{2}(f(a)+f(b))-\frac{(b-a)^{3} f^{\prime \prime}(\eta)}{12} \\
=\frac{b-a}{12}(f(a)+f(b))+E, \quad \eta \in[a, b],
\end{gathered}
$$

where $E$ is error of integration. Suppose $t_{i}=\frac{i}{m}=i h$ and $I_{i}=\left[t_{i-1}, t_{i}\right]$. The representation error when $f(t)$ is represented by a series of BPFs over every subinterval $\left[t_{i}, t_{i}+\frac{h}{k}\right], i=$ $0, \ldots, m-1$ is

$$
e_{i}(t)=f(t)-f_{i} \psi_{i}(t)=f(t)-f_{i},
$$

where $f_{i}=\frac{1}{h} \int_{i h}^{(i+1) h} f(t) d t$. From $(7)$

$$
f_{i}=\frac{1}{2}\left(f\left(t_{i}\right)+f\left(t_{i}+h\right)\right)+E .
$$

It is obvious that if $f(t)=C$ (constant), then $e_{i}(t)=0$. So, this error is computed for $f(t)=t$ in interval $\left[t_{i}, t_{i}+\frac{h}{k}\right], i=1, \ldots, m-1$. 
For this function $E=0$, so

$$
e_{i}(t)_{\left[t_{i}, t_{i}+\frac{h}{k}\right]}=\left|t-f_{i}\right|=\left|t-\frac{t_{i}+t_{i+1}}{2}\right|=\left|t-\left(t_{i}+\frac{h}{2}\right)\right| \leq \frac{h}{2},
$$

then this error with BPFs is $\frac{h}{2} M$.

Similarly, the error when $f(t)$ is represented in a series of $\varepsilon$ MBPFs over every subinterval $\left[t_{i}, t_{i}+\frac{h}{k}\right]$ is

$$
\begin{aligned}
e_{i}(t)_{\left[t_{i}, t_{i}+\frac{h}{k}\right]} & =\left|t-\left(\frac{\sum_{j=0}^{k-1}\left(t_{i}-\left(\frac{j h}{k}\right)+t_{i+1}-\left(\frac{j h}{k}\right)\right)}{2 k}\right)\right| \\
& =\left|t-\left(\frac{\sum_{j=0}^{k-1}\left(t_{i}-\left(\frac{j h}{k}\right)+t_{i}+h-\left(\frac{j h}{k}\right)\right)}{2 k}\right)\right| \\
& =\left|t-\left(t_{i}+\frac{h}{2}\right)-\frac{(k-1) h}{2 k}\right| \\
& \leq \frac{h}{2 k} .
\end{aligned}
$$

So, the error with $\varepsilon$ MBPFs is $\frac{h}{2 k} M$.

For $I_{0}$ in $\left[0, \frac{h}{k}\right]$ we have

$$
\begin{aligned}
e_{i}(t)_{\left[0, \frac{h}{k}\right]} & =\left|t-\sum_{j=0}^{k-1} \frac{h-\left(\frac{j h}{k}\right)}{2 k}\right| \\
& =\left|t-\left(\frac{h}{2}-\frac{(k-1) h}{4 k}\right)\right| \\
& =\left|t-\left(\frac{h}{4}+\frac{h}{4 k}\right)\right| \\
& =\mathcal{O}\left(\frac{h}{4}\right)
\end{aligned}
$$

So, the error is $\mathcal{O}\left(\frac{h}{4}\right)$ also for $I_{n}$ 
Now,

$$
\begin{aligned}
\left\|e_{i}(t)\right\|^{2} & =\int_{t_{i}}^{t_{i}+\frac{h}{k}}\left|e_{i}(t)\right|^{2} d t \\
& =\int_{t_{i}}^{t_{i}+\frac{h}{k}} \frac{h^{2}}{4 k^{2}} M^{2} d t \\
& =\frac{h^{3}}{4 k^{3}} M^{2} \\
\left\|e_{i}\right\|^{2} & =\int_{0}^{1} e^{2}(t) d t \\
& =\int_{0}^{1}\left(\sum_{i=1}^{m} \sum_{j=0}^{k-1} e_{i}(t)\right)^{2} d t \\
& =\sum_{i=1}^{m} \sum_{j=0}^{k-1} \int_{0}^{1} e_{i}^{2}(t) d t \\
& =\sum_{i=1}^{m} \sum_{j=0}^{k-1}\left\|e_{i}(t)\right\|^{2} \\
& =\frac{1}{h} \cdot k \cdot \frac{h^{3}}{4 k^{3}} M^{2} \\
& =\frac{h^{2}}{4 k^{2}} M^{2} .
\end{aligned}
$$

We define the representation error between $f(s, t)$ and its 2D- $\varepsilon$ MBPFs expansion $f_{i j}$ over every subregion $D_{i j}$, is defined as

$$
e_{i j}(s, t)=f(s, t)-f_{i j},
$$

where $D_{i j}:=\left\{(s, t) \mid t_{i} \leq s \leq t_{i}+\frac{h}{k}, t_{j} \leq t \leq t_{j}+\frac{h}{k}\right\}$.

Based on Taylors expansion and similarity to the above discussion,

$$
\|e(s, t)\|=\frac{h}{2 k} M \text {. }
$$

Theorem 3.3. Assume that

$i P(\omega \in \Omega:\|u(\omega, t)\|<C)=1$

ii $\left\|k_{i}\right\|<C, i=1,2$.

Then

$$
\begin{gathered}
\sup \left(E(\|u-\bar{u}\|)^{2}\right)^{\frac{1}{2}}=\mathcal{O}\left(\frac{h}{k}\right), t \in[h, 1-h] . \\
0 \leq t \leq T
\end{gathered}
$$

Proof. For a complete proof see [6].

\subsection{Examples of linear Volterra-Fredholm integral equations.}

Example 3.4. consider the linear Volterra-Fredholm integral equation

$$
f(t)=1-\int_{0}^{t}(t-s) f(s) d s+\int_{0}^{\pi} f(s) d s,
$$

with the exact solution is $f(t)=\cos t$. 
TABLE 1. The exact and approximate solution of Example 3.4 for $m=257$

\begin{tabular}{|c|c|c|}
\hline$t$ & Exact & Approximation \\
\hline 0 & 1 & 1.004 \\
\hline 0.3 & 0.9553 & 0.958 \\
\hline 0.6 & 0.8254 & 0.8274 \\
\hline 0.9 & 0.6216 & 0.6234 \\
\hline 1.2 & 0.362 & 0.3641 \\
\hline 1.5 & 0.07053 & 0.07265 \\
\hline 1.8 & -0.2268 & -0.2256 \\
\hline 2.1 & -0.5046 & -0.5035 \\
\hline 2.4 & -0.7373 & -0.7365 \\
\hline 2.7 & -0.9043 & -0.9049 \\
\hline 3 & -0.99 & -0.9927 \\
\hline
\end{tabular}

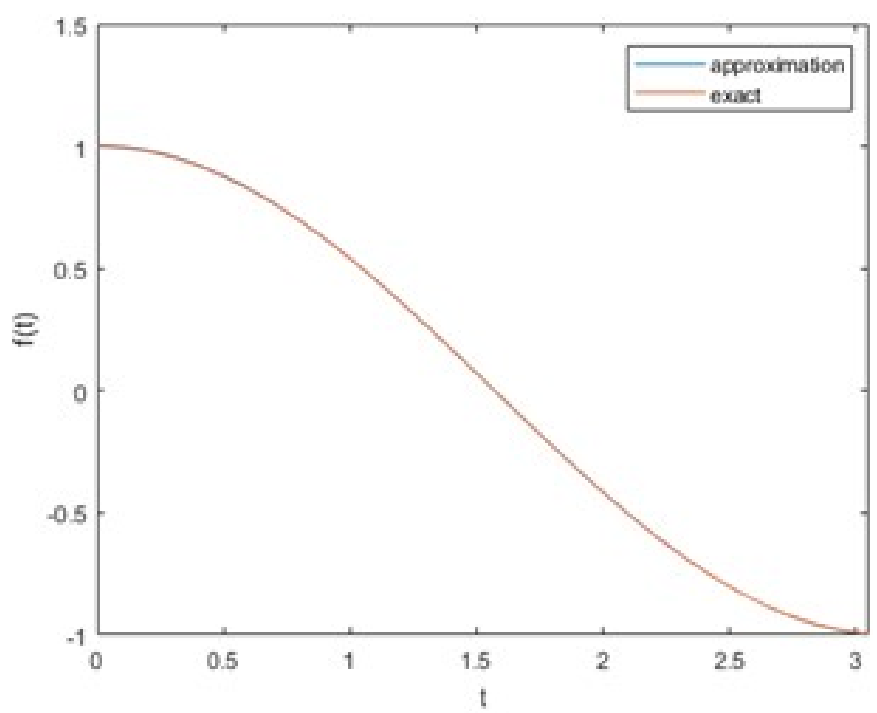

FIGURE 1. The trajectory of the exact and approximation solution of Example 3.4

Example 3.5. consider the linear Volterra-Fredholm integral equation

$$
f(t)=t-2 e^{t}+e^{-t}+1+\int_{0}^{t} s e^{t} f(s) d s+\int_{0}^{1} e^{s+t} f(s) d s,
$$

with the exact solution is $f(t)=e^{-t}$. 
TABLE 2. The exact and approximate solution of Example 3.5 for $m=257$

\begin{tabular}{|c|c|c|}
\hline$t$ & Exact & Approximation \\
\hline 0 & 1 & 0.9991 \\
\hline 0.1 & 0.9048 & 0.9017 \\
\hline 0.2 & 0.8187 & 0.8162 \\
\hline 0.3 & 0.7408 & 0.7388 \\
\hline 0.4 & 0.6704 & 0.6688 \\
\hline 0.5 & 0.6065 & 0.6054 \\
\hline 0.6 & 0.5488 & 0.548 \\
\hline 0.7 & 0.4966 & 0.496 \\
\hline 0.8 & 0.4493 & 0.449 \\
\hline 0.9 & 0.4066 & 0.4064 \\
\hline 1 & 0.3679 & 0.3683 \\
\hline
\end{tabular}

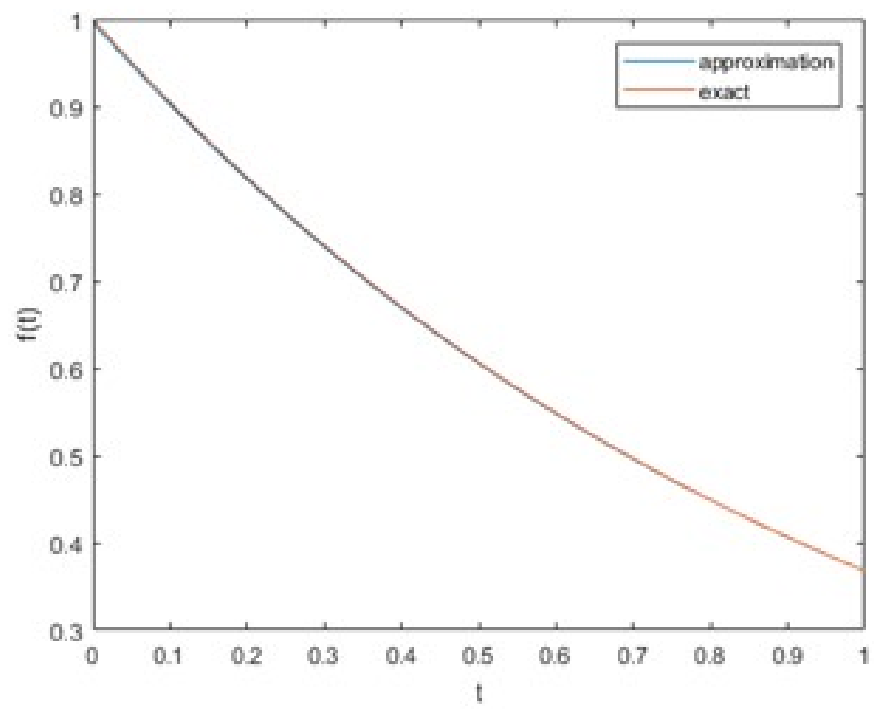

FiguRE 2. The trajectory of the exact and approximation solution of Example 3.5

\section{Conclusions}

The $\varepsilon$ MBPFs and their integration operational matrix are used to obtain the solution of linear Volterra-Fredholm integral equations. The present method reduces a linear VolterraFredholm integral equations into a system of algebraic equations. The convergence and error analysis of the proposed method are investigated. Some numerical examples are given, we plot approximate and exact solution to demonstrate the efficiency and accuracy of the proposed method. The results show that the approximate solutions of the proposed method have a good of efficiency and accuracy. 


\section{REFERENCES}

[1] Dastjerdi, H. L. and F. M. Maalek Ghaini, 2012, Numerical Solution of Volterra-Fredholm Integral Equations by Moving Least Square Method and Chebyshev Polynomials. Applied Mathematical Modelling, $36(7): 3283-3288$

[2] Hendi, F. A. and A. M. Albugami, 2010, Numerical Solution for Fredholm-Volterra Integral Equation of the Second Kind by Using Collocation and Galerkin Methods. Journal of King Saud University (Science), $22(1): 37-40$.

[3] Jiang, Z. H. and W. Schaufelberger, 1992, Block Pulse Functions and Their Applications in Control Systems. Springer.

[4] Kanwal, Ram P., 1997, Linear Integral Equations Theory 83 Technique, Second Edition. Springer Science+Business Media.

[5] Maleknejad, K., H. Almasieh and M. Roodaki, 2010, Triangular Functions (TF) Method for the Solution of Nonlinear Volterra-Fredholm Integral Equations.Communications in Nonlinear Science and Numerical Simulation, 15(11):3293-3298.

[6] Maleknejad, K., M. Khodabin, and F. Hosseini Shekarabi, 2014, Modified Block Pulse Functions for Numerical Solution of Stochastic Volterra Integral Equations. Journal of Applied Mathematics, 4:1-10.

[7] Nemati, S., 2015, Numerical Solution of Volterra-Fredholm Integral Equations Using Legendre Collocation Method. Journal of Computational and Applied Mathematics, 278:29-36.

[8] Rahman, M., 2007, Integral Equations and Their Applications. WIT Press.

[9] Tang, Xiaojun., 2015, Numerical Solution of Volterra-Fredholm Integral Equations Using Parameterized Pseudospectral Integration Matrices. Applied Mathematics and Computation, 270(1):744-755.

[10] Wazwaz, Abdul-Majid., 2011, Linear and Nonlinear Integral Equations (Methods and Applications), Higher Education Press and Springer-Verlag Berlin Heidelberg.

[11] Wazwaz, Abdul-Majid., 2015, A First Course in Integral Equations, Second Edition, World Scientific.

[12] Yalcinbas, Salih., 2002, Taylor Polynomial Solutions of Nonlinear Volterra-Fredholm Integral Equations. Applied Mathematics and Computation, 127(2-3):195-206. 